\title{
Gender-Specific Association of Galanin Polymorphisms with HPA-Axis Dysregulation, Symptom Severity, and Antidepressant Treatment Response
}

\author{
Paul G Unschuld*,', Marcus Ising', Darina Roeske', Angelika Erhardt', Michael Specht', Stefan Kloiber', \\ Manfred Uhr', Bertram Müller-Myhsok', Florian Holsboer' and Elisabeth B Binder' \\ 'Max Planck Institute of Psychiatry, Kraepelinstrasse, München, Germany
}

\begin{abstract}
Galanin (GAL) is an estrogen-inducible neuropeptide, highly expressed in brain regions reported to be involved in regulation of mood and anxiety. GAL possibly has a direct modulatory effect on hypothalamic-pituitary-adrenal (HPA)-axis regulation. Recent data from pharmacological and genetic studies indicate a significant function of GAL in stress-related disorders. By using a tag SNP approach covering the locus encoding preprogalanin (PPGAL), earlier findings of female-specific associations of polymorphisms in this locus with panic disorder were expanded to a larger sample of 268 outpatients with anxiety disorders (ADs). Within a larger sample of 54I inpatients with major depressive disorder (MDD), we then tested associations of one PPGAL tag SNP with specific depression symptom clusters and HPA-axis activity assessed by the combined dexamethasone-suppression/CRH-stimulation test both at inpatient admission and discharge $(n=298)$. Gender specificity as well as dependence of the association on levels of circulating estrogens was analyzed. Genotyping revealed high linkage disequilibrium in the promoter area of the PPGAL gene, which includes several estrogen-response elements. Confirming earlier results, rs948854, tagging this promoter region, was associated with more severe anxiety pathology in female AD patients, but not in males. In premenopausal female MDD patients, the same allele of rs948854 was associated with more severe vegetative but not cognitive depressive symptoms at discharge and worse treatment response on antidepressant medication. Furthermore, this allele was associated with higher HPA-axis activity at admission. No significant case-control associations could be observed. However, because of power limitations of both patient samples, small effects cannot be excluded. The reported associations in independent samples of AD and MDD support an estrogen-dependent function of GAL in pathophysiology of anxiety and depression, affecting response to antidepressant treatment.

Neuropsychopharmacology (2010) 35, I583-1592; doi: I0.1038/npp.2010.30; published online 17 March 2010
\end{abstract}

Keywords: galanin; association; genetics; anxiety; depression; response

\section{INTRODUCTION}

Human galanin (GAL) is a 30 amino-acid neuropeptide, proteolytically processed from preprogalanin (PPGAL) (Evans and Shine, 1991; Schmidt et al, 1991). PPGAL is a single-copy gene located on chromosome 11q13.3-13.5, spanning over $6 \mathrm{~kb}$ of genomic DNA and organized into six exons (Rokaeus and Brownstein, 1986; Vrontakis et al, 1987). Estrogen-response elements (ERE) in the promoter region have been proposed to be responsible for estrogeninduced regulation of GAL expression (Howard et al, 1997; Kaplan et al, 1988). For $G A L$, so far three G-protein-coupled receptors (galanin receptor 1 (GALR1), GALR2, GALR3)

*Correspondence: Dr PG Unschuld, Max Planck Institute for Psychiatry, Kraepelinstrasse 2-10, München, Bavaria 80804, Germany, Tel: + 49893062 2324, Fax: + 49893062 2605,

E-mail: unschuld@mpipsykl.mpg.de

Received 15 September 2009; revised 23 January 2010; accepted 16 February 2010 have been described and are expressed in both the central and peripheral nervous system (reviewed in Mitsukawa et al, 2008). Genetic associations of $P P G A L$ and the genes encoding GALR3 have been reported for psychiatric phenotypes (Belfer et al, 2006, 2007; Levran et al, 2008; Unschuld et al, 2008). In animal studies, GAL has been shown to modulate anxious and depressive behavior (reviewed in Kuteeva et al, 2008). Intraperitoneal application of a nonpeptide GAL receptor agonist showed antidepressant-like effects in rats (Bartfai et al, 2004) and the intravenous application of $G A L$ has been reported to have an antidepressant effect in patients with depression under standard antidepressant treatment (Murck et al, 2004).

In human beings, besides being expressed in anxietyrelevant brain regions such as the locus coeruleus (LC) and amygdala, particularly high concentrations for GAL have been reported for the hypothalamus and the pituitary (Gentleman et al, 1989; Michener et al, 1990), with strong 
evidence for a regulatory function of $G A L$ in the control of anterior pituitary function (Lopez et al, 1991; Ottlecz et al, 1988). The regulatory effect of $G A L$ on neuroendocrine stress reactions and its involvement in anxious and depressive symptomatology have been thoroughly reviewed (Kuteeva et al, 2008; Mechenthaler, 2008; Picciotto, 2008; Wrenn and Crawley, 2001). It has been hypothesized that GAL affects stress-related behavior by interacting not only with monoaminergic neurotransmitters such as serotonin (5-HT) and noradrenaline, but also with $\gamma$-aminobutyric acid (Fuxe et al, 1998; Kehr et al, 2002; Khoshbouei et al, 2002a; Ma et al, 2001; Sharkey et al, 2008; Tyszkiewicz et al, 2008; Xu et al, 1998). GAL expression in the LC and limbic nuclei may be stimulated by stress (Holmes et al, 1995; O'Neal et al, 2001; Sweerts et al, 1999) and seems to have a significant impact on hypothalamic-pituitary-adrenal (HPA)-axis signaling (Brogan et al, 1999; Khoshbouei et al, 2002b; Lopez et al, 1991; Malendowicz et al, 1994). In preclinical and clinical studies, corticotropin-releasing hormone (CRH) and vasopressin (AVP) have been shown to be the main effectors of HPA activity, important for the mediation of anxious and depressive-like behavior (Britton et al, 1986; Holsboer, 2000; Landgraf, 2006; Nemeroff and Owens, 2002; Pepin et al, 1992; Stenzel-Poore et al, 1994; Strohle et al, 1998). There is both an anatomical and functional connection between $G A L$ and the CRH/AVP system, as some hypothalamic neurons in the paraventricular nucleus co-express these peptides (Arvat et al, 1995; Ceccatelli et al, 1989; Leibowitz, 1998; Mazzocchi et al, 1992) and GAL inhibits stress-induced ACTH secretion, presumably by altering $\mathrm{CRH}$ and/or AVP release from nerve terminals in the median eminence (Hooi et al, 1990).

This concatenation of data thus suggests that GAL may be important in anxiety- and depression-related behavior and also HPA-axis regulation. In an earlier study, we could show a gender-specific association of PPGAL-promoter polymorphisms with severity of anxious pathology in a small sample of female panic-disorder patients (Unschuld et al, 2008). The aim of this study was to expand our initial finding in female panic-disorder patients using an increased patient sample with panic disorder as well as other anxiety disorders (ADs) and a sample of patients with major depressive disorder (MDD). As our initial report identified female gender-specific associations and EREs have been identified in the PPGAL-promoter area, we hypothesized that the female-specific effects in our earlier report (Unschuld et al, 2008) might be related to differences in circulating estrogen levels and, therefore, stratified our analyses not only by gender, but also menopause status, which was possible in the larger MDD sample. Owing to the observed genetic associations with anxious depression in this analysis and the reported worse antidepressant treatment response in patients with anxious depression (Fava et al, 2009), we also tested whether PPGAL polymorphisms would associate with treatment response, using the Hamilton depression (HAMD)-rating scale. Finally, we examined whether menopause-status-specific associations can also be observed on the neuroendocrine level, using the combined dexamethasone-suppression/CRH-stimulation (Dex-CRH) test. As described above, interactions of the GAL system on the HPA-axis have been reported.

\section{MATERIALS AND METHODS}

\section{Study Population}

This study protocol was approved by the local ethics committee and is in compliance with the Code of Ethics of the World Medical Association (Declaration of Helsinki). Only individuals over 18 years were included, written informed consent was obtained from all participating subjects. Ethnicity was recorded using a self-reported sheet for perceived nationality, language, and ethnicity of the subject itself, parents, and grandparents. The level of anxiety and depression symptoms is described in Table 1.

\section{AD Patients}

The AD-outpatient sample included 268 individuals $(62 \%$ females, $80 \%$ of the females $<46$ years), recruited at their first visit with the following ADs at the Max-PlanckInstitute of Psychiatry (MPI): panic disorder with agoraphobia $(71.6 \%)$, panic disorder without agoraphobia (12.3\%), and social phobia (8.1\%), agoraphobia (2.4\%), specific phobia (3.3\%), and generalized AD (2.4\%). These include the 121 panic-disorder patients analyzed in Unschuld et al (2008). All patients underwent a clinical examination including EEG and ECG. Exclusion criteria were ADs in the context of substance abuse, a medical or neurological disorder and a concurrent major depressive, bipolar, or axis II disorder. Affective and anxious pathology was assessed at the patient's first presentation using HAMD-rating scales (Hamilton, 1960) (Table 1). As a

Table I Statistics of the Analyzed Samples

\begin{tabular}{|c|c|c|c|}
\hline \multirow[t]{2}{*}{ Sample } & \multirow{2}{*}{$\begin{array}{l}\text { All patients } \\
\text { Mean (SD) }\end{array}$} & \multirow{2}{*}{$\begin{array}{l}\text { Females } \\
\text { Mean (SD) }\end{array}$} & \multirow{2}{*}{$\frac{\text { Males }}{\text { Mean (SD) }}$} \\
\hline & & & \\
\hline \multicolumn{4}{|l|}{$A D$} \\
\hline Age & 38.27 (12.09) & $39.02(12.78)$ & $37.05(10.83)$ \\
\hline Age at onset & $27.24(11.42)$ & $27.65(11.80)$ & $26.64(10.82)$ \\
\hline HAMD & | $3.92(6.6)$ & | 3.64 (6.69) & $14.39(6.46)$ \\
\hline PAS B & $6.86(3.05)$ & $7.06(2.9)$ & $6.54(3.27)$ \\
\hline
\end{tabular}

$\begin{array}{lccc}\text { MDD } & & & \\ \text { Age } & 48.38(\mid 3.88) & 48.75(\mid 3.96) & 47.99(\mid 3.84) \\ \text { Age at onset } & 36.83(\mid 5.62) & 35.53(\mid 5.46) & 38.20(\text { I5.7I) } \\ \text { HAMD adm. } & 22.52(8.89) & 23.15(9.42) & 21.9(8.34) \\ \text { HAMD dis. } & 7.82(5.88) & 8.39(6.09) & 7.27(5.63) \\ \text { Veget. anxiety adm. } & 2.09(0.75) & 2.18(0.8 \mid) & 1.99(0.67) \\ \text { Veget. anxiety dis. } & 0.68(0.54) & 0.71(0.57) & 0.65(0.5 \mid)\end{array}$

Controls

$\begin{array}{cccc}\text { Age } & 47.43(13.46) & 47.76(14.1) & 47.04(12.66) \\ \text { BDI } & 3.74(3.78) & 3.78(3.70) & 3.68(3.88)\end{array}$

'adm.' stands for test scores at admission and 'dis.' for discharge.

Hamilton depression-rating scale (HAMD, 21 items, Hamilton, 1960), Panic and Agoraphobia subscale B (PAS B, Bandelow, 1995), vegetative and cognitive depression subscales (Rhoades and Overall, 1983), Beck Depression Inventory (BDI, Beck et al, |96|). 
measure of severity of anxious pathology, indicating particularly phobic avoidance and impairment of life quality, the Bandelow-panic and agoraphobia subscale B (PAS-B) was used (Bandelow, 1995). In our earlier study, a different scale was used, assessing severity of panic attacks (Unschuld et al, 2008). A total of $89 \%$ of the $\mathrm{AD}$ patients were German Caucasians, other nationalities were Central European countries and the United States (with German Caucasian ancestry).

\section{MDD Patients}

The MDD-patient sample consisted of 541 patients (59\% females, $44 \%$ of the females $<46$ years), all inpatients at the MPI of Psychiatry with the diagnosis of unipolar depression (25.9\% first episode, $74.1 \%$ recurrent) and participants of the MARS study (Hennings et al, 2009). All patients were included within the first 3 days of their stay in this study and underwent clinical examination including EEG and ECG. Exclusion criteria were known manic or hypomanic episodes, mood incongruent psychotic symptoms, substance abuse, and a major internal medical or neurological disorder. Affective pathology was assessed using HAMDrating scales, 21 item scale (Hamilton, 1960), and further characterized using the factorial HAMD subscales for cognitive (HAMDcog) and vegetative depression (HAMDveg) established by Rhoades and Overall (1983). Psychometric assessments were performed at inpatient admission and discharge, at the time points at which the combined Dex-CRH test was used for analysis (Table 1). Time from admission to psychometric assessment was used as a covariate in association calculations. In addition, for each patient completing the study, the individual difference of overall HAMD at admission and discharge was calculated (HAMDdiff). An HAMD score at discharge below 10 was used as a threshold for remission as described earlier (Ising et al, 2009). During their hospital stay, all MDD inpatients were treated according to the doctor's choice using a combination of psychopharmacological and cognitivebehavioral therapy efforts. Antidepressant treatment at the time point of the initial Dex-CRH test was mainly monotherapy $(56.2 \%)$, of which $25.1 \%$ were on selective serotonin re-uptake inhibitors, $12.1 \%$ on tricyclic antidepressants, and $14.7 \%$ on Mirtazapine as the three largest groups. A total of $8.1 \%$ of the patients did initially not receive psychopharmacological treatment; $42.2 \%$ concomitantly received benzodiazepines, $2.1 \%$ mood-stabilizers (except Carbamazepine), and 3.7\% neuroleptic medication. An earlier study had shown that number and length of ineffective antidepressant treatment trials before hospitalization and type of medication at the time point of the test other than Carbamazepine did not influence the endocrine response in the Dex-CRH test at admission (Kunzel et al, 2003).

\section{Healthy Controls}

The control sample used in this study consisted of 541 individuals (52\% females), who were matched for ethnicity, sex, and age to the MDD sample. All controls were screened for the absence of $\mathrm{ADs}$, depression, or substance dependence using the Composite International Diagnostic
Screener (Wittchen, 1999), and current depressive symptomatology was recorded using the Beck Depression Inventory (Beck et al, 1961) (Table 1). Only individuals without any severe neurological or medical disorder and axis I disorder were included in the study. A total of $91.7 \%$ were German Caucasians, other nationalities were Central European countries and the United States with German Caucasian ancestry.

\section{Dex-CRH Test}

The Dex-CRH test was performed as described in detail earlier (Heuser et al, 1994b). Patients were administered the test within the first 10 days of admission and the last 10 days of discharge. Briefly, patients were orally pre-treated with $1.5 \mathrm{mg}$ of dexamethasone at $23: 00 \mathrm{~h}$. The following day a venous catheter was placed at 1430 hours and blood was drawn at 1500,1530,1545, 1600, and 1615 hours into tubes containing EDTA and Trasylol (Bayer, Germany). At 1502 hours, $100 \mu \mathrm{g}$ of human CRH (Ferring, Kiel, Germany) was administered IV. For the area under the curve (AUC) of the cortisol (CAUC) and ACTH (AAUC) response, the area under the concentration-time course curve was computed using a trapezoidal integration for the test at admission as well as at discharge. Time from admission to the first Dex$\mathrm{CRH}$ test was used as a covariate in association calculations; however, there was no correlation with CAUC and AAUC at admission $(r=-0.024, p=0.63$ and $r=-0.031, p=0.52$, respectively) and no significant difference between genders.

Dex-CRH testing was performed in 298 MDD patients (53\% females), at a mean of $6.04(\mathrm{SD}=2.15)$ days after admission, and 245 of these patients additionally in the last week before discharge, after a mean hospital stay of 11.7 $(\mathrm{SD}=8.6)$ weeks.

\section{Grouping of Female MDD Patients According to Menopausal Status}

Women $<46$ years at the time point of the Dex-CRH test were categorized as high-estrogen premenopausal and women $>50$ years as low-estrogen postmenopausal. This categorization concurs with earlier reports of incidence of menopause in western industrialized countries (Matthews et al, 1989). Women aged between 46 and 49 years were excluded from these analyses. In a subgroup of patients, plasma sex-hormone levels were assayed for samples taken at the time of the admission Dex-CRH test and subdivided in premenopausal and postmenopausal women. Estrogen levels were significantly higher, LH and follicle-stimulating hormone (FSH) levels lower in premenopausal $(n=28)$ than in postmenopausal $(n=27)$ MDD inpatients (mean (SEM): estradiol (pg/ml) 69.32 (11.81) vs 27.83 (4.63), $p=0.01$; FSH $(\mu \mathrm{U} / \mathrm{ml}) 20.93$ (7.07) vs 70.03 (10.19), $p=0.001 ; \mathrm{LH}(\mu \mathrm{U} / \mathrm{ml})$ 10.53 (2.33) vs 30.82 (4.68), $p=0.002$ ).

\section{Hormone Assays}

Briefly, for the measurement of plasma cortisol concentrations, a radioimmunoassay kit (ICN Biomedicals, Carson, $\mathrm{CA}$ ) was used in which the detection limit was $0.3 \mathrm{ng} / \mathrm{ml}$. For plasma ACTH concentrations, an immunometric assay without extraction (Nichols Institute, San Juan Capistrano, 
CA) was used with a detection limit of $4.0 \mathrm{pg} / \mathrm{ml}$. In 59 female MDD -patients additionally to Dex-CRH testing, plasma estradiol, luteinizing hormone ( $\mathrm{LH})$, and folliclestimulating hormone (FSH) concentrations were measured by commercially available ELISA. The intraassay variations were between 5.6 and $8.2 \%$ and the interassay variations were in the range of $6.6-8.7 \%$.

\section{DNA Preparation, SNP Selection, and Genotyping}

On enrollment in the study, $40 \mathrm{ml}$ of EDTA blood was drawn from each subject and DNA was instantly extracted using a standardized procedure (Puregene whole blood DNA-extraction kit; Gentra Systems, MN). Six SNPs (rs2187331, rs1893679, rs3136540, rs3136541, rs1042577, and rs6591350) genotyped using the Illumina Sentrix Human-1 Genotyping 100K and 300K BeadChip systems (Illumina, San Diego, CA) are located within the PPGAL locus and were included in the analysis. The SNP rs948854, which is not included in the HapMap CEU dataset and thus the Illumina arrays, was assessed using the MALDI-TOF Sequenom (Sequenom, San Diego, CA) genotyping platform as described earlier (Binder et al, 2004). The rs948854 was included as it had shown associations with the tested phenotypes in an earlier report of our group (Unschuld et al, 2008). These seven SNPs located within the PPGAL locus and $5.6 \mathrm{~kb} 5^{\prime}$ flanking region were genotyped in 541 healthy controls. The call rate for each SNP was $>99.6 \%$ (mean 99.9\%), and minor allele frequencies all exceeded $15 \%$. No SNP showed a significant deviation $(p<0.05)$ from Hardy-Weinberg equilibrium. SNP genotyping was performed at the Center for Applied GenoTyping, Munich, Germany. The program HaploView 4.0 (Barrett et al, 2005) was applied to determine linkage disequilibrium (LD). By using a cut off for $r^{2}>0.8$, three tag SNPs (rs2187331, rs948854, and rs1042577) covering PPGAL were identified (Figure 1) and selected for the genetic analyses to be performed in $268 \mathrm{AD}$ patients. These three selected SNPs cover $100 \%$ of the genetic variation described in HapMap version II in the region chr11:68 202 000-68216000 at $r^{\wedge} 2$ $>0.8$ (cut off for minor allele frequencies $>0.05$, http:// www.hapmap.org, Consortium, 2003) in the Caucasian CEU population. As rs948854 is not included in the HapMap dataset, the adjacent polymorphism rs1893679, which is predicted in our control sample by $\mathrm{rs} 948854$ at $\mathrm{r}^{\wedge} 20.95$, was used together with the tag-SNPs rs2187331 and rs1042577 to calculate the genetic coverage of the three tag SNPs for the $P P G A L$ region in the CEU population.

\section{Statistical Data Analysis}

Statistical analyses were performed using the WG-Permer software, a tool for rapid analysis of large-scale genomeassociation studies (Ripke, 2007, http://www.wg-permer. org) and the commercial SPSS-statistics package, version 16.0 (2007 SPSS inc.). As the AD-patient sample was-in contrast to the MD sample - not matched for age and sex with the control sample, age and sex were considered as covariates in the case-control analyses by using logistic regression. In the MDD samples, genetic association with psychopathology and the cortisol and ACTH response in the Dex-CRH test were also analyzed stratified by gender and

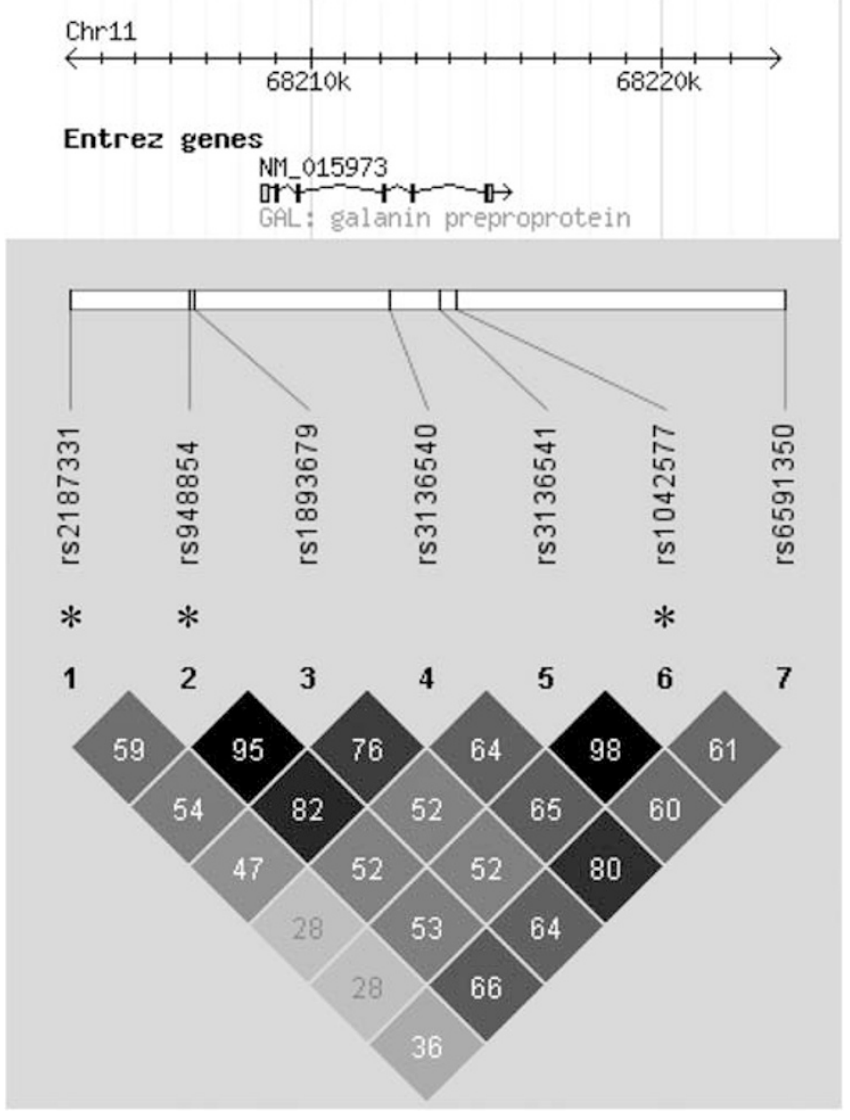

Figure I LD chart of the analyzed SNPs within the locus encoding GAL (PPGAL), displaying linkage of the particular markers $\left(r^{\wedge} 2\right)$ calculated using HaploView 4.0 (Barrett et al, 2005), with an MAF cut off at 5\% and an $r^{\wedge} 2$ cut off $>0.8$. Tagging SNPs are marked with '*.'

presumed menopausal status. This resulted in three subgroups of MDD patients ( $n$-counts: psychopathology at admission; at discharge; Dex-CRH test performed): premenopausal women $(18-45$ years; $n=102 ; 91 ; 61)$, postmenopausal women $(50-80$ years; $n=150 ; 134 ; 81)$ and male MDD patients $(n=221 ; 200 ; 140)$. The three groups did not significantly differ in basal cortisol levels or CAUC and AAUC, indicating no effect of gender as described earlier (Hennings et al, 2009). There were also no significant differences in any of the psychometric measures.

For the analysis of LD and selection of tagging SNPs, the program HaploView 4.0 (http://www.broad.mit.edu/mpg/ haploview, Barrett et al, 2005) was used. The association analysis for SNPs and phenotypes was conducted by applying allelic and carrier-frequency models. The phenotypes describing severity of anxious- and depressive pathologies (PAS-B, HAMD) showed normal distribution in the analyzed samples. However, to increase robustness of the analysis, permutation-based estimates of $p$-values were used in patients and controls. The minimum $\mathrm{P}$ method of Westfall and Young (WY) with 100000 permutations was used to correct for multiple testing of tag SNP- and phenotype associations (Westfall and Young, 1993) both in case-control and dimensional analyses, taking into account possible correlation between predictors. The 
$p$-value adjustment was performed as follows in the particular samples:

(1) Tests for case-control associations in the AD and MDD samples were adjusted for testing of three tag SNPs in three genetic models (allelic, carriers of the frequent, and rare alleles, respectively) according to WY (Westfall and Young, 1993).

(2) Tests for PAS-B associations in the AD sample were adjusted for SNPs and three genetic models, according to WY (Westfall and Young, 1993).

(3) For analyses in the MDD sample, only rs948854 was analyzed by applying solely an allelic model of genetic testing. Adjustment of $p$-values for multiple testing was performed using Bonferroni correction (Bland and Altman, 1995) for the number of tests:

(a) Interaction terms on HAMD were corrected for testing pre- and posttreatment values. Interaction terms on Dex/CRH response were corrected for testing pre- and posttreatment and additionally ACTH and cortisol.

(b) Consecutive exploratory tests for significant HAMD interactions were corrected for testing the subcategories of assumed estrogen status (premenopausal $v s$ postmenopausal and males) and additionally testing both overall HAMD and HAMDdiff, its subcategories vegetative HAMD, and cognitive HAMD. In the consecutive exploratory tests for significant Dex-CRH test interactions, nominal $p$-values were adjusted for testing the subcategories of assumed estrogen status and the laboratory values cortisol and ACTH.

The level of significance for all statistical analyses was set to $5 \%$ after correction for multiple testing.

Calculations of power to detect case-control associations in the $\mathrm{AD}$ patient and $\mathrm{MDD}$ sample, respectively, were performed using the program Quanto, Version 1.2.4 (http:// hydra.usc.edu/gxe/), with a level of significance 0.006 (correcting for SNPs and three models of genetic testing), disease allele frequency of $>0.15$, an additive genetic model and disease prevalences as reported in the US National Comorbidity Survey Replication (Kessler et al, 2005). Power to detect a genotype relative risk of 1.7 (additive model) in the $\mathrm{AD}$ sample was $88 \%$ and in the MDD sample $98 \%$. Power to detect a female-specific genotype relative risk of 1.7 in the AD sample was $63 \%$ and $82 \%$ in the MDD sample.

Information about predicted promoter positions, geneexon boundaries, and LD in other populations was retrieved from publicly accessible online databases (http://www. genecards.org, http://www.genome.cse.ucsc.edu, http:// www.hapmap.org, http://www.snpper.chip.org).

\section{RESULTS}

\section{Associations with Severity of Female AD: Expansion} of Results Presented in Unschuld et al (2008)

In this expanded AD sample, we could support the earlier reported association of severity of anxiety symptoms with PPGAL SNPs in female patients only. The rs948854, but not the two other tag-SNPs, showed gender-specific association with anxiety severity as measured using the PAS-B subscale.
Female carriers of the rare G-allele of rs948854 showed significantly higher PAS-B scores, when compared with carriers of the more frequent A-allele (PAS-B rs948854 G-allele: 7.6, SE 0.28 and A-allele: 6.86, SE 0.20, allelic model: nominal $p=0.008, p<0.05$ after correction for multiple testing; carrier-A and carrier-G models not significant after correction for multiple testing). When analyzed separately, carriers of the G-allele of rs948854 show higher severity measures in both the female panic patients reported in our earlier publication (cohort 1) (PAS-B means (SEM): A:6.75(0.42); G:7.54(0.42)) and the newly recruited female patients with different ADs (cohort 2) ((PAS-B means (SEM) A:6.12(0.62); G:7.81(0.41)). No association of severity could be observed for male patients when an allelic and carrier model was applied. Stratification by menopausal status was not performed, because of the low $\mathrm{N}$ in the postmenopausal group and thus power limitations.

\section{Dependence of Association with Anxiety Severity in Female Patients on Menopausal Status: Evidence from an MDD Sample}

We then tested whether the G-allele of rs948854 was also associated with depression or anxiety symptom severity at admission or discharge in the MDD sample stratified by gender and menopausal status, as this was possible in the larger MDD sample. Using an ANCOVA with HAMD as outcome and rs948854 allele, group (pre-, $v s$ postmenopausal women or male) and interaction of SNP by group as predictors, and time from admission to discharge as covariate, we observed a significant interaction between SNP and group for HAMD at discharge, but not at admission (HAMD at discharge, allelic model: nominal $p=0.001, \mathrm{~F}_{1,849}=10.26, p<0.01$ after correction for multiple testing). There were no significant main effects of the rs948854 allele status or patient group.

Secondary exploratory testing revealed that at discharge, premenopausal female MDD inpatients showed a significantly higher HAMD score in carriers of the G-allele of rs948854 compared with carriers of the A-allele (11.55, SE 1.28 vs 7.34, SE 0.56, allelic model: nominal $p=0.002$; $p<0.05$ after correction for multiple testing) and worse response (HAMDdiff, means(SEM) G: 16.16(1.41); A: 19.31(74), nominal $p=0.040$ ). The percentage of patients with HAMD score at discharge below 10 as a threshold for remission differed in the subgroup of premenopausal patients depending on rs948854 allele status (95\% CI): A: 61\% (48.25-72.44); G: $40.25 \%$ (32.94-48.02). OR for premenopausal carriers of the rs948854 G-allele for nonremission at discharge was 2.3 (95\% CI: 1.26-4.29; nominal $p=0.006$ ). This may be a reflection of higher vegetative anxious-, but not cognitive depressive symptoms, as analyzed using the HAMD subscales described above. Premenopausal female carriers of the G-allele of rs 948854 showed nominally significant higher values for the vegetative depression subscale, than carriers of the frequent allele (HAMDveg: 0.94 SE 0.15 and 0.61 SE 0.06, allelic model: nominal $p=0.039$ ), but no significant difference in the subscale cognitive depression. These differences were neither observed in postmenopausal women nor in male patients (Figure 2). 

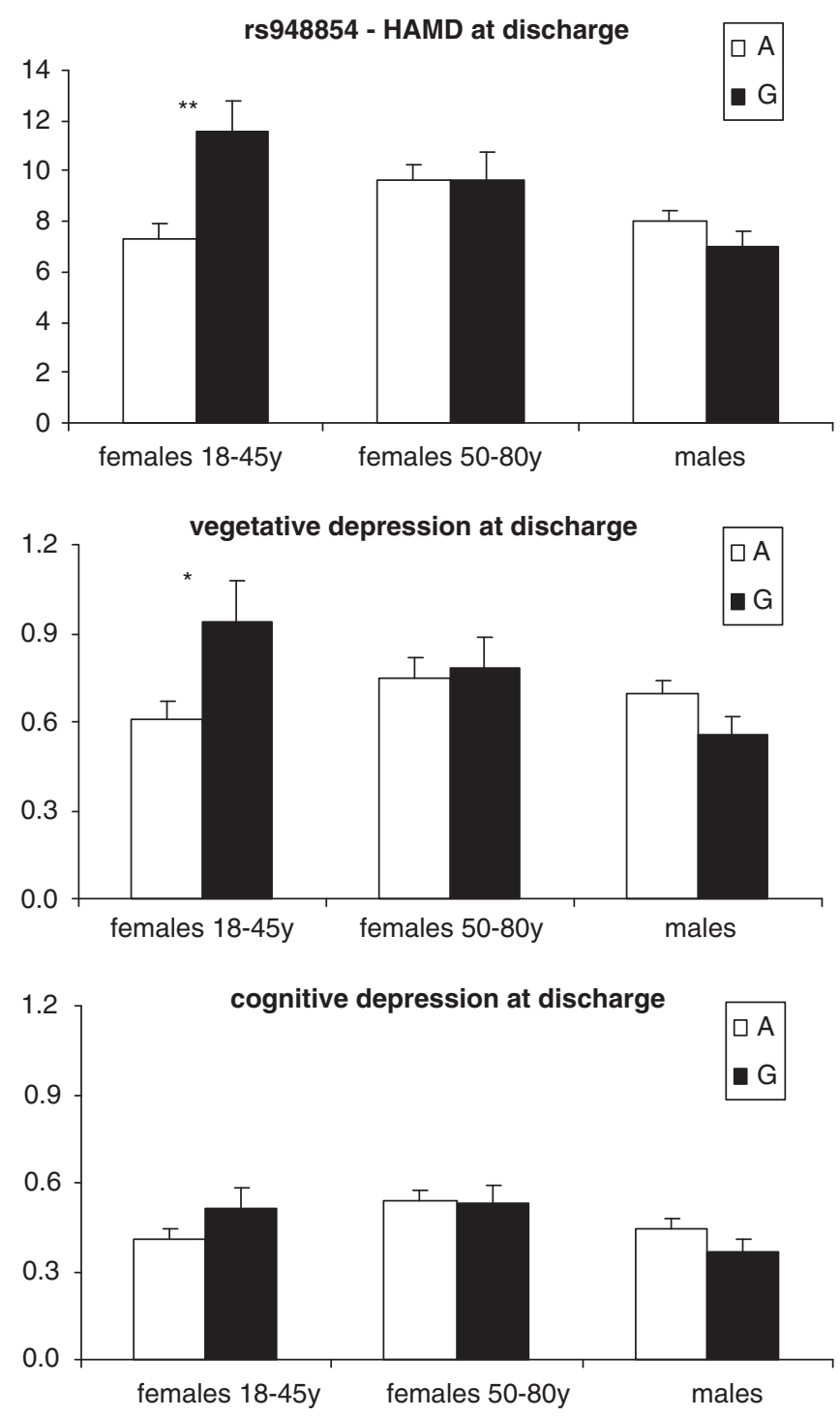

Figure 2 The rs948854 allele distributions for HAMD 21 items (Hamilton, 1960), and the subscales vegetative and cognitive depression (Rhoades and Overall, 1983) at discharge. Associations significant after correction for multiple testing are labeled with '***, nominal significance with '*.'

\section{Associations of rs948854 with HPA-Axis Activity in Premenopausal MDD}

Subsequently, we tested whether the G-allele of rs 948854 was associated with the endocrine response in the Dex-CRH test in any of the three MDD-patient groups. An ANCOVA was used with either CAUC or AAUC as outcome and rs948854 allele, group (pre-, vs postmenopausal women or male) and interaction of SNP by group as predictors, and time from admission to test as covariate. We observed a significant interaction between SNP and group for AAUC and CAUC at admission, but not at discharge (CAUC, allelic model: nominal $p=0.014, \mathrm{~F}_{1,559}=6.14, p<0.05$ after correction for multiple testing; AAUC, allelic model: nominal $p=0.020, \mathrm{~F}_{1,559}=5.47$, not significant after correction for multiple testing). There were no significant main effects of the rs948854 allele status or patient group.
Secondary exploratory tests revealed that carriers of the G-allele of rs948854 in the premenopausal women subgroup had significantly higher cortisol responses in the Dex-CRH test (CAUC rs948854: 4825.35, SE 876.15 and 3223.74, SE 320.67 , nominal $p=0.005$, allelic model; $p<0.05$ after correction for multiple testing). ACTH response in the premenopausal women subgroup was significantly higher for carriers of the rare allele of the SNP rs948854 (AAUC rs948854: 1568.70, SE 190.68 and 1139.93, SE 71.79, nominal $p=0.001$, allelic model; $p<0.01$ after correction for multiple testing) (Figure 3).

\section{Case-Control Associations}

Using allelic and carrier models, no significant case-control associations could be observed for the $\mathrm{AD}$ sample and the MDD sample as a whole or stratified by gender after correction for multiple testing for any of the three tag SNPs.

\section{DISCUSSION}

Expanding earlier findings of our research group on singlelocus and haplotype effects, including in particular associations of the rare allele of the PPGAL SNP rs948854 with more severe pathology in female panic-disorder patients (Unschuld et al, 2008), now a more than doubled sample of patients with ADs was analyzed using a tag SNP approach covering the genetic locus encoding GAL and $5.6 \mathrm{~kb}$ of its $5^{\prime}$ promoter region (Figure 1). Consistent with the earlier findings, the rare G-allele of rs 948854 was associated with more severe anxious pathology in female, but not male patients. Severity of anxiety symptoms in general was measured using the PAS-B scale, which reflects phobic avoidance and resulting every-day limitations of the patients (Bandelow, 1995), whereas in our earlier study, a panic-specific version of this scale was used (Unschuld et al, 2008). No case-control association could be observed; however, our $\mathrm{AD}$ sample lacked power to detect smaller risks, especially if only tested in women.

As $G A L$ has been implicated in the pathophysiology of anxiety as well as depression (reviewed in Kuteeva et al, 2008; Mechenthaler, 2008; Picciotto, 2008; Wrenn and Crawley, 2001) and because anxiety symptoms are common in patients with MDD (Fava et al, 2009), this study was extended to an MDD-inpatient sample. The use of this larger sample also allowed to stratify the analyses by menopausal status, as we hypothesized that the genderspecific genetic associations observed in the $\mathrm{AD}$ sample were driven by circulating estrogen levels. Functional EREs have been described in the promoter region of $P P G A L$ (Howard et al, 1997; Kaplan et al, 1988). In these analyses, we could show that association of symptom severity was specific to the group of premenopausal women. At inpatient discharge, premenopausal MDD patients, carriers of the rare G-allele of rs948854, showed a significantly higher symptom severity as measured by the HAMD and less remission after antidepressant therapy (OR 2.3, 95\% CI: 1.26-4.29). Analysis of HAMD subscales specific for vegetative $v s$ cognitive symptoms revealed that this difference was presumably carried by an enhanced vegetative but not cognitive depressive pathology with the 

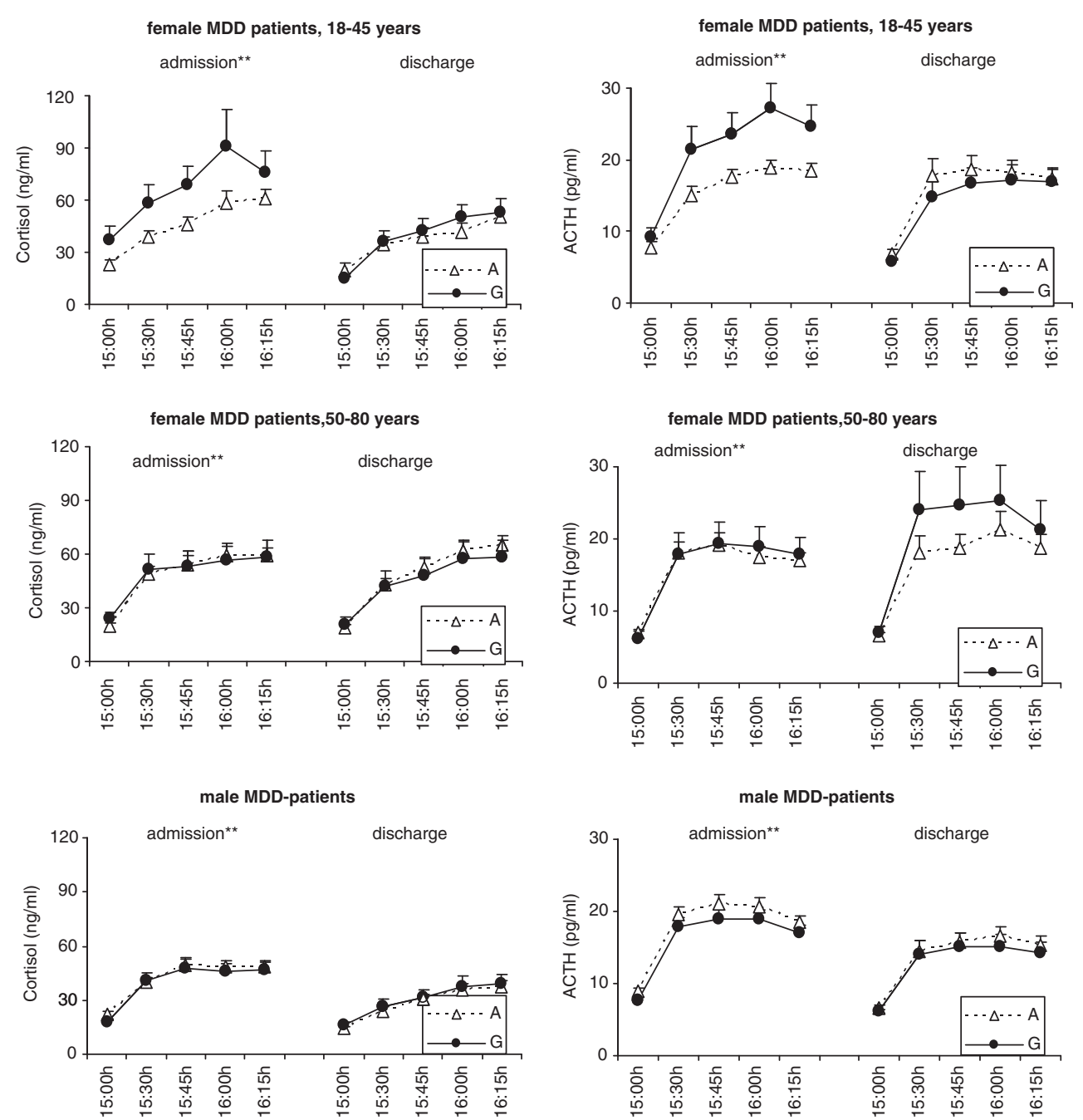

Figure 3 The rs948854-dependent levels of Cortisol (ng/ml) and ACTH (pg/ml) measured at admission and discharge of MDD inpatients, displayed separately for the analyzed subsamples: premenopausal women, postmenopausal women, and males. At I 502 hours, IV administration of I $00 \mu \mathrm{g}$ CRH was performed, the 1530 hours measurement of cortisol and ACTH was the first after stimulation. Solely in the subgroup of premenopausal women at admission, significant allele-dependent differences between cortisol and ACTH levels after stimulation could be observed.

rs948854 G-allele. The direction of the association was thus similar to the one observed for the AD sample, with more anxious pathology in female G-allele carriers (Figure 2). Vegetative depressive symptoms correlate more closely with anxiety symptoms in depression than cognitive symptoms (Rhoades and Overall, 1983). In a study analyzing symptom stability in recurrent major depression, anxious pathology measured according to the subscales defined by Overall and Rhoades appeared as a particular robust category in terms of symptom stability across episodes and might indicate an anxious subtype of depressive disorder (Oquendo et al, 2004). It has been speculated earlier that anxious depression might depict a subcategory of MDD, which shows particularly poor response to antidepressive therapy (Fava et al, 2009; Ising et al, 2009; Nemeroff and Owens, 2002) and may be consistent with our findings of high vegetative symptoms and worse overall treatment response at discharge in premenopausal MDD patients, carrying the G-allele of rs948854.
Case-control associations were negative for MDD as well as AD. However, it has to be taken into account that group sizes of both samples are small for case-control studies, especially when stratified by gender, thus lacking power to detect small effects. In addition, a number of phenotypes have been tested in the $\mathrm{AD}$ and MDD sample. When correcting for multiple testing as described in Materials and methods section, the associations in female AD patients with severity and in MDD premenopausal women with HAMD at discharge, cortisol, and ACTH response at admission remain significant. The associations of the G-allele of rs 948854 with the vegetative HAMD subscale at discharge and HAMD improvement after therapy in premenopausal women only show nominal significance, not withstanding correction for multiple testing, so that these associations need to be interpreted with caution. Misclassification of menopausal status based on age may have reduced power of this study, as individual conditions of altered estrogen levels or irregular incidence of 
menopause are possible unnoticed confounders. However, to minimize effects of misclassification, patients who reported hormone replacement therapy or known medical disorders influencing hormone status were excluded from analyses.

As described in the introduction, $G A L$ is also involved in HPA-axis regulation. To explore whether the observed genetic and psychopathological associations are also reflected by an impaired stress hormone regulation, we analyzed the association of rs 948854 with plasma cortisol and ACTH response in the Dex-CRH test. We show that the G-allele of this SNP was associated with enhanced plasma cortisol and ACTH response at admission only in premenopausal women with MDD. This might indicate an enhanced dysfunction of HPA-axis regulation in these G-allele carriers also showing enhanced anxiety and vegetative symptoms, which might be a reflection of increased CRH and/or AVP signaling. These two neuropeptides have been linked to anxiety symptoms as well as HPAaxis activation in both human beings (Bardeleben and Holsboer, 1989, 2000; Heuser et al, 1994a; Nemeroff and Owens, 2002; Strohle et al, 1998) and animals (Britton et al, 1986; Keck et al, 2002; Landgraf, 2006), and are coexpressed in neurons with GAL (Arvat et al, 1995; Ceccatelli et al, 1989; Leibowitz, 1998; Mazzocchi et al, 1992).

The tag-SNP rs948854 captures the genetic variation within a $6.2 \mathrm{~kb}$ region starting at $-1780 \mathrm{bp} 5^{\prime}$ from the $P P G A L$ gene (Figure 1). This region includes the so-far reported EREs at positions $-1162,-527,-365,-361,-122$ (Howard et al, 1997; Kaplan et al, 1988; Kofler et al, 1995). The rs948854 might thus either directly influence binding and activation of the EREs or be in LD with such a functional variant.

In rodents, estrogen has been shown to significantly induce $G A L$ expression in behaviorally relevant loci such as hypothalamus, pituitary, and LC (Brann et al, 1993; Hemmer and Hyde, 1992; Kaplan et al, 1988; Tseng et al, 1997). In human beings, significantly higher GAL serum levels have been measured in premenopausal than in postmenopausal women (Meczekalski et al, 2001). The rs948854 might alter the function of EREs in the PPGAL promoter and thus the induction of GAL by estrogens. As anxiogenic as well as anxiolytic effects of $G A L$ have been reported (reviewed in Kuteeva et al, 2008), it is difficult to predict whether the G-allele of this SNP is associated with enhanced or reduced GAL induction by estrogen. Possibly estrogens can also moderate the described stress-related induction of $G A L$, providing an additional mechanism for the gender and menopause-status-dependent SNP associations.

$G A L$ has been suggested earlier to modulate the release of $\mathrm{CRH}$ and/or AVP, as it attenuates stress-induced ACTH secretion (Hooi et al, 1990). Stress-related increases in CRH and AVP secretion may be counteracted by GAL and thereby lead to differences in anxious behavior and HPAaxis activation in the two genetic-promoter variants. The rs948854 likely influences estrogen-driven $G A L$ induction and by that possibly stifle CRH and AVP activation.

The reported associations of PPGAL polymorphisms in two samples of $\mathrm{AD}$ and MDD patients with severity of anxiety symptoms and HPA-axis activity thus support a function of $G A L$ and its receptors in the pathophysiology of anxiety and depression and show the importance of genderand hormone-status-specific genetic associations.

\section{ACKNOWLEDGEMENTS}

We thank Sabine Damast, Maik Koedel, and Susann Sauer for excellent technical assistance. This work has been funded by the Bavarian Ministry of Commerce and by the Federal Ministry of Education and Research (BMBF) in the framework of the National Genome Research Network (NGFN), Förderkennzeichen 01GS0481. We are responsible for the contents of this publication.

\section{DISCLOSURE}

All the authors, except FH and EBB, declare that, except for the income received from our primary employers, no financial support or compensation has been received from any individual or corporate entity over the past 3 years for research or professional service and there are no personal financial holdings that could be perceived as constituting a potential conflict of interests. FH wishes to declare that he personally received grant support from Bristol Myer Squibb, is founder and share holder of Affectis, and share holder of Corcept and Neurocrine. EBB wishes to declare that she personally received grant support from the National Institute of Mental Health and the Doris Duke Charitable foundation.

\section{REFERENCES}

Arvat E, Gianotti L, Ramunni J, Grottoli S, Brossa PC, Bertagna A et al (1995). Effect of galanin on basal and stimulated secretion of prolactin, gonadotropins, thyrotropin, adrenocorticotropin and cortisol in humans. Eur J Endocrinol 133: 300-304.

Bandelow B (1995). Assessing the efficacy of treatments for panic disorder and agoraphobia. II. The Panic and Agoraphobia Scale. Int Clin Psychopharmacol 10: 73-81.

Bardeleben U, Holsboer F (1989). Cortisol response to a combined dexamethasone-human corticotrophin-releasing hormone challenge in patients with depression. J Neuroendocrinol 1: 485-488.

Barrett JC, Fry B, Maller J, Daly MJ (2005). Haploview: analysis and visualization of LD and haplotype maps. Bioinformatics 21: 263-265.

Bartfai T, Lu X, Badie-Mahdavi H, Barr AM, Mazarati A, Hua XY et al (2004). Galmic, a nonpeptide galanin receptor agonist, affects behaviors in seizure, pain, and forced-swim tests. Proc Natl Acad Sci USA 101: 10470-10475.

Beck AT, Ward CH, Mendelson M, Mock J, Erbaugh J (1961). An inventory for measuring depression. Arch Gen Psychiatry 4: 561-571.

Belfer I, Hipp H, Bollettino A, McKnight C, Evans C, Virkkunen M et al (2007). Alcoholism is associated with GALR3 but not two other galanin receptor genes. Genes Brain Behav 6: 473-481.

Belfer I, Hipp H, McKnight C, Evans C, Buzas B, Bollettino A et al (2006). Association of galanin haplotypes with alcoholism and anxiety in two ethnically distinct populations. Mol Psychiatry 11: 301-311.

Binder EB, Salyakina D, Lichtner P, Wochnik GM, Ising M, Putz B et al (2004). Polymorphisms in FKBP5 are associated with increased recurrence of depressive episodes and rapid response to antidepressant treatment. Nat Genet 36: 1319-1325.

Bland JM, Altman DG (1995). Multiple significance tests: the Bonferroni method. BMJ 310: 170. 
Brann DW, Chorich LP, Mahesh VB (1993). Effect of progesterone on galanin mRNA levels in the hypothalamus and the pituitary: correlation with the gonadotropin surge. Neuroendocrinology 58: 531-538.

Britton KT, Lee G, Dana R, Risch SC, Koob GF (1986). Activating and 'anxiogenic' effects of corticotropin releasing factor are not inhibited by blockade of the pituitary-adrenal system with dexamethasone. Life Sci 39: 1281-1286.

Brogan RS, Coney LK, Wehrenberg WB, Beretta G, Giustina A (1999). Short-term glucocorticoid administration decreases both hypothalamic and pituitary galanin synthesis in the adult male rat. Metabolism 48: 792-796.

Ceccatelli S, Eriksson M, Hokfelt T (1989). Distribution and coexistence of corticotropin-releasing factor-, neurotensin-, enkephalin-, cholecystokinin-, galanin- and vasoactive intestinal polypeptide/peptide histidine isoleucine-like peptides in the parvocellular part of the paraventricular nucleus. Neuroendocrinology 49: 309-323.

Consortium TIH (2003). The International HapMap project. Nature 426: 789-796.

Evans HF, Shine J (1991). Human galanin: molecular cloning reveals a unique structure. Endocrinology 129: 1682-1684.

Fava M, Hwang I, Rush AJ, Sampson N, Walters EE, Kessler RC (2009). The importance of irritability as a symptom of major depressive disorder: results from the national comorbidity survey replication. Mol Psychiatry (originally published online 10 March 2009; doi:10.1038/MP.2009.20).

Fuxe K, Jansson A, Diaz-Cabiale Z, Andersson A, Tinner B, Finnman UB et al (1998). Galanin modulates 5-hydroxytryptamine functions. Focus on galanin and galanin fragment/5hydroxytryptamine1A receptor interactions in the brain. Ann $N$ Y Acad Sci 863: 274-290.

Gentleman SM, Falkai P, Bogerts B, Herrero MT, Polak JM, Roberts GW (1989). Distribution of galanin-like immunoreactivity in the human brain. Brain Res 505: 311-315.

Hamilton M (1960). A rating scale for depression. J Neurol Neurosurg Psychiatry 23: 56-62.

Hemmer A, Hyde JF (1992). Regulation of galanin secretion from pituitary cells in vitro by estradiol and GHRH. Peptides 13: 1201-1206.

Hennings JM, Owashi T, Binder EB, Horstmann S, Menke A, Kloiber S et al (2009). Clinical characteristics and treatment outcome in a representative sample of depressed inpatientsfindings from the Munich Antidepressant Response Signature (MARS) project. J Psychiatr Res 43: 215-229.

Heuser I, Yassouridis A, Holsboer F (1994). The combined dexamethasone/CRH test: a refined laboratory test for psychiatric disorders. J Psychiatr Res 28: 341-356.

Holmes PV, Blanchard DC, Blanchard RJ, Brady LS, Crawley JN (1995). Chronic social stress increases levels of preprogalanin mRNA in the rat locus coeruleus. Pharmacol Biochem Behav 50: 655-660.

Holsboer F (2000). The corticosteroid receptor hypothesis of depression. Neuropsychopharmacology 23: 477-501.

Hooi SC, Maiter DM, Martin JB, Koenig JI (1990). Galaninergic mechanisms are involved in the regulation of corticotropin and thyrotropin secretion in the rat. Endocrinology 127: 2281-2289.

Howard G, Peng L, Hyde JF (1997). An estrogen receptor binding site within the human galanin gene. Endocrinology 138: 4649-4656.

Ising M, Lucae S, Binder EB, Bettecken T, Uhr M, Ripke S et al (2009). A genome-wide association study points to multiple loci predicting antidepressant treatment outcome in depression. Arch Gen Psychiatry 66: 966-975.

Kaplan LM, Gabriel SM, Koenig JI, Sunday ME, Spindel ER, Martin JB et al (1988). Galanin is an estrogen-inducible, secretory product of the rat anterior pituitary. Proc Natl Acad Sci USA 85: 7408-7412.
Keck ME, Wigger A, Welt T, Muller MB, Gesing A, Reul JM et al (2002). Vasopressin mediates the response of the combined dexamethasone/CRH test in hyper-anxious rats: implications for pathogenesis of affective disorders. Neuropsychopharmacology 26: 94-105.

Kehr J, Yoshitake T, Wang FH, Razani H, Gimenez-Llort L, Jansson A et al (2002). Galanin is a potent in vivo modulator of mesencephalic serotonergic neurotransmission. Neuropsychopharmacology 27: 341-356.

Kessler RC, Chiu WT, Demler O, Merikangas KR, Walters EE (2005). Prevalence, severity, and comorbidity of 12-month DSMIV disorders in the National Comorbidity Survey Replication. Arch Gen Psychiatry 62: 617-627.

Khoshbouei H, Cecchi M, Dove S, Javors M, Morilak DA (2002a). Behavioral reactivity to stress: amplification of stress-induced noradrenergic activation elicits a galanin-mediated anxiolytic effect in central amygdala. Pharmacol Biochem Behav 71: 407-417.

Khoshbouei H, Cecchi M, Morilak DA (2002b). Modulatory effects of galanin in the lateral bed nucleus of the stria terminalis on behavioral and neuroendocrine responses to acute stress. Neuropsychopharmacology 27: 25-34.

Kofler B, Evans HF, Liu ML, Falls V, Iismaa TP, Shine J et al (1995). Characterization of the $5^{\prime}$-flanking region of the human preprogalanin gene. DNA Cell Biol 14: 321-329.

Kunzel HE, Binder EB, Nickel T, Ising M, Fuchs B, Majer M et al (2003). Pharmacological and nonpharmacological factors influencing hypothalamic-pituitary-adrenocortical axis reactivity in acutely depressed psychiatric in-patients, measured by the DexCRH test. Neuropsychopharmacology 28: 2169-2178.

Kuteeva E, Hokfelt T, Wardi T, Ogren SO (2008). Galanin, galanin receptor subtypes and depression-like behaviour. Cell Mol Life Sci 65: 1854-1863.

Landgraf $\mathrm{R}$ (2006). The involvement of the vasopressin system in stress-related disorders. CNS Neurol Disord Drug Targets 5: 167-179.

Leibowitz SF (1998). Differential functions of hypothalamic galanin cell grows in the regulation of eating and body weight. Ann N Y Acad Sci 863: 206-220.

Levran O, Londono D, O'Hara K, Nielsen DA, Peles E, Rotrosen J et al (2008). Genetic susceptibility to heroin addiction; a candidate-gene association study. Genes Brain Behav 7: 720-729.

Lopez FJ, Merchenthaler I, Ching M, Wisniewski MG, Negro-Vilar A (1991). Galanin: a hypothalamic-hypophysiotropic hormone modulating reproductive functions. Proc Natl Acad Sci USA 88: $4508-4512$.

Ma X, Tong YG, Schmidt R, Brown W, Payza K, Hodzic L et al (2001). Effects of galanin receptor agonists on locus coeruleus neurons. Brain Res 919: 169-174.

Malendowicz LK, Nussdorfer GG, Nowak KW, Mazzocchi G (1994). The possible involvement of galanin in the modulation of the function of rat pituitary-adrenocortical axis under basal and stressful conditions. Endocr Res 20: 307-317.

Matthews KA, Meilahn E, Kuller LH, Kelsey SF, Caggiula AW, Wing RR (1989). Menopause and risk factors for coronary heart disease. $N$ Engl J Med 321: 641-646.

Mazzocchi G, Malendowicz LK, Rebuffat P, Nussdorfer GG (1992). Effects of galanin on the secretory activity of the rat adrenal cortex: in vivo and in vitro studies. Res Exp Med (Berl) 192: 373-381.

Mechenthaler I (2008). Galanin and the neuroendocrine axes. Cell Mol Life Sci 65: 1826-1835.

Meczekalski B, Slopien R, Warenik-Szymankiewicz A (2001). The concentration of galanin in postmenopausal women before and after application of hormone replacement therapy compared to women of reproductive age. Med Sci Monit 7: 928-932. 
Michener SR, Aimone LD, Yaksh TL, Go VL (1990). Distribution of galanin-like immunoreactivity in the pig, rat and human central nervous system. Peptides 11: 1217-1223.

Mitsukawa K, Lu X, Bartfai T (2008). Galanin, galanin receptors and drug targets. Cell Mol Life Sci 65: 1796-1805.

Murck H, Held K, Ziegenbein M, Kunzel H, Holsboer F, Steiger A (2004). Intravenous administration of the neuropeptide galanin has fast antidepressant efficacy and affects the sleep EEG. Psychoneuroendocrinology 29: 1205-1211.

Nemeroff CB, Owens MJ (2002). Treatment of mood disorders. Nat Neurosci 5(Suppl): 1068-1070.

O’Neal HA, Van Hoomissen JD, Holmes PV, Dishman RK (2001). Prepro-galanin messenger RNA levels are increased in rat locus coeruleus after treadmill exercise training. Neurosci Lett 299: 69-72.

Oquendo MA, Barrera A, Ellis SP, Li S, Burke AK, Grunebaum M et al (2004). Instability of symptoms in recurrent major depression: a prospective study. Am J Psychiatry 161: 255-261.

Ottlecz A, Snyder GD, McCann SM (1988). Regulatory role of galanin in control of hypothalamic-anterior pituitary function. Proc Natl Acad Sci USA 85: 9861-9865.

Pepin MC, Pothier F, Barden N (1992). Impaired type II glucocorticoid-receptor function in mice bearing antisense RNA transgene. Nature 355: 725-728.

Picciotto MR (2008). Galanin and addiction. Cell Mol Life Sci 65: 1872-1879.

Rhoades HM, Overall JE (1983). The Hamilton Depression Scale: factor scoring and profile classification. Psychopharmacol Bull 19: 91-96.

Ripke S (2007). WG-Permer: Rapid Analysis of Large Scale Whole Genome Association Studies. MPI of Psychiatry: Munich. http://www.wg-permer.org.

Rokaeus A, Brownstein MJ (1986). Construction of a porcine adrenal medullary cDNA library and nucleotide sequence analysis of two clones encoding a galanin precursor. Proc Natl Acad Sci USA 83: 6287-6291.

Schmidt WE, Kratzin H, Eckart K, Drevs D, Mundkowski G, Clemens A et al (1991). Isolation and primary structure of pituitary human galanin, a 30-residue nonamidated neuropeptide. Proc Natl Acad Sci USA 88: 11435-11439.
Sharkey LM, Madamba SG, Siggins GR, Bartfai T (2008). Galanin alters GABAergic neurotransmission in the dorsal raphe nucleus. Neurochem Res 33: 285-291.

Stenzel-Poore MP, Heinrichs SC, Rivest S, Koob GF, Vale WW (1994). Overproduction of corticotropin-releasing factor in transgenic mice: a genetic model of anxiogenic behavior. J Neurosci 14(5 Pt 1): 2579-2584.

Strohle A, Poettig M, Barden N, Holsboer F, Montkowski A (1998). Age- and stimulus-dependent changes in anxiety-related behaviour of transgenic mice with GR dysfunction. Neuroreport 9: 2099-2102.

Sweerts BW, Jarrott B, Lawrence AJ (1999). Expression of preprogalanin mRNA following acute and chronic restraint stress in brains of normotensive and hypertensive rats. Brain Res Mol Brain Res 69: 113-123.

Tseng JY, Kolb PE, Raskind MA, Miller MA (1997). Estrogen regulates galanin but not tyrosine hydroxylase gene expression in the rat locus ceruleus. Brain Res Mol Brain Res 50: 100-106.

Tyszkiewicz JP, Fong TM, Dong Y (2008). GABAB receptors are required for galanin modulation of membrane properties of neurons in the arcuate nucleus of rats. Brain Res 1191: 63-68.

Unschuld PG, Ising M, Erhardt A, Lucae S, Kohli M, Kloiber S et al (2008). Polymorphisms in the galanin gene are associated with symptom-severity in female patients suffering from panic disorder. J Affect Disord 105: 177-184.

Vrontakis ME, Peden LM, Duckworth ML, Friesen HG (1987). Isolation and characterization of a complementary DNA (galanin) clone from estrogen-induced pituitary tumor messenger RNA. J Biol Chem 262: 16755-16758.

Westfall PH, Young SS (1993). Resampling-based Multiple Testing: Examples and Methods for p-value Adjustment. Wiley: New York.

Wittchen HU (1999). Screening for mental disorders: performance of the Composite International Diagnostic-Screener (CID-S). Int J Methods Psychiatr Res 8: 59-70.

Wrenn CC, Crawley JN (2001). Pharmacological evidence supporting a role for galanin in cognition and affect. Prog Neuropsychopharmacol Biol Psychiatry 25: 283-299.

Xu ZQ, Zhang X, Pieribone VA, Grillner S, Hokfelt T (1998). Galanin-5-hydroxytryptamine interactions: electrophysiological, immunohistochemical and in situ hybridization studies on rat dorsal raphe neurons with a note on galanin R1 and R2 receptors. Neuroscience 87: 79-94. 